\title{
HOLOMORPHIC MAPPINGS BETWEEN BALLS AND POLYDISCS
}

\author{
R. R. SIMHA
}

Abstract. A proof based only on Schwarz's lemma is given for the fact that the polydisc and the ball are not holomorphically equivalent.

1. The purpose of this note is to record the following consequence of Schwarz's lemma:

Proposition A. Let B and $P$ denote the unit ball and the unit polydisc in $\mathbf{C}^{n}$ respectively, with the origin as centre. Then for any holomorphic map $f: B \rightarrow P$ (respectively, $g: P \rightarrow B$ ) preserving the origin, $d f(0)$ (respectively, $d g(0)$ ) maps $B$ into $P$ (respectively, $P$ into $B$ ).

Proposition A immediately implies the classical

Proposition B. For $n \geqslant 2, B$ and $P$ are not holomorphically equivalent.

Proposition A is implicit in Carathéodory [2, pp.790-791].

2. Proof of Proposition B. Since $P$ is evidently homogeneous, we need only show that there is no isomorphism between $B$ and $P$ which preserves the origin. But if such an isomorphism existed, then by Proposition $A$ its differential at the origin would be a linear isomorphism between $B$ and $P$. However, it is obvious that, for $n \geqslant 2, B$ and $P$ are not linearly isomorphic, since the boundary of $P$ contains linear pieces of positive dimension, whereas the boundary of $B$ does not.

3. Proof of Proposition A. Let $f: B \rightarrow P$ be a holomorphic map with $f(0)=0$. If $f=\left(f_{1}, \ldots, f_{n}\right)$, we must show that, for $u=\left(u_{1}, \ldots, u_{n}\right) \in B$, $\left|\sum_{i=1}^{n} u_{i} D_{i} f_{j}(0)\right| \leqslant 1$ for every $j$. But this follows from Schwarz's lemma, which is applicable to the function $t \mapsto f_{j}\left(u_{1} t, \ldots, u_{n} t\right)=f_{j}(t u)$ for any $u \in B$.

Now let $g: P \rightarrow B$ be any holomorphic map with $g(0)=0$. We must show that $d g(0)(v) \in B$ for all $v \in P$. Now, for any $u \in B$, Schwarz's lemma can be applied to the function $t \mapsto \sum u_{i} g_{i}(t v)$ and yields $\langle\bar{u}, d g(0)(v)\rangle \mid \leqslant 1$. Hence, $d g(0)(v) \in B$ as required.

4. It is well known that, if $n \geqslant 2$, the polydisc $P \subset \mathbf{C}^{n}$ cannot be mapped properly into a ball in any $\mathbf{C}^{m}$. For a quick proof of this fact, as well as references to related matters, see Alexander [1, Remark, p.255].

5. With the notation of Proposition A, we see from Proposition A and Hadamard's determinantal inequality [3, p.175] that $|\operatorname{det} d f(0)| \leqslant 1$ and that

Received by the editors December 30, 1974.

AMS (MOS) subject classifications (1970). Primary 32A07; Secondary 30A42. 
$\mid$ det $d g(0) \mid<1$ if $n \geqslant 2$. This again implies Proposition B. Actually, even the sharp estimate $|\operatorname{det} d g(0)| \leqslant(1 / \sqrt{ } n)^{n}$ (cf. [2, p.791]) follows in the same way if one observes that the distinguished boundary of the polydisc of radius $1 / \sqrt{ } n$ in $\mathbf{C}^{n}$ contains an orthonormal basis for $\mathbf{C}^{n}$, e.g., the vectors $e_{k}=\left(e_{k o}, \ldots\right.$, $\left.e_{k, n-1}\right)$ where $e_{k l}=n^{-1 / 2} \exp (2 \pi i k l / n), 0 \leqslant k, l \leqslant n-1$.

6. We have formulated Proposition A as above because we wanted an elementary proof for Proposition B. But what our argument proves is the following (notation: Banach space means complex Banach space, and for any Banach space $E, U(E)$ is the open unit ball).

Proposition C. Let E,F be Banach spaces, and $h: U(E) \rightarrow U(F)$ a holomorphic map with $h(0)=0$. Then $\|d h(0)\| \leqslant 1$.

Proof. Let $v \in U(E)$ and $\lambda \in U\left(F^{*}\right)$. Then Schwarz's lemma applied to the function $t \mapsto \lambda(h(t v))$ yields $|\lambda(h(t v))| \leqslant|t|$. Hence $\|h(t v)\| \leqslant|t|$ by HahnBanach, so $d h(0)(v)=\lim _{t \rightarrow 0} h(t v) t^{-1}$ has norm at the most one. Q.E.D.

COROLlary. If $h$ is a holomorphic equivalence, $d h(0)$ is a linear isometry between $E$ and $F$.

Proposition D. Let $E$ be a Banach space, and $h: U(E) \rightarrow U(E)$ a holomorphic map with $h(0)=0$. Then if dh(0) is the identity, $h$ is the identity.

Proof. Let $v \in E$ have norm one. Then for any $\lambda \in U\left(E^{*}\right)$ with $\lambda(v)=1$ (there exist such $\lambda$ by Hahn-Banach), Schwarz's lemma applies to the function $t \mapsto \lambda(h(t v))$ and yields $\lambda(h(t v)) \equiv t$, or $\lambda(h(t v)-t v)=0$. So, again by HahnBanach, $h(t v)=t v$. Q.E.D.

COROLLARY. Any origin-preserving holomorphic equivalence of unit balls of Banach spaces is a linear isometry.

In particular, the only origin-preserving automorphisms of balls, polydiscs, etc. in $\mathbf{C}^{n}$ are the obvious linear ones.

Finally, I thank the referee for drawing my attention to the results of [1].

\section{REFERENCES}

1. H. Alexander, Holomorphic mappings from the ball and polydisc, Math. Ann. 209 (1974), 249-256.

2. C. Carathéodory, Uber die Abbildungen, die durch Systeme von analytischen Funktionen von mehreren Veränderlichen erzeugt werden, Math. Z. 34 (1932), 758-792.

3. F. Riesz and B. Sz.-Nagy, Leçons d'analyse fonctionnelle, 4th ed., Gauthier-Villars, Paris, 1965; English transl. of 2nd ed., Ungar, 1955. MR 17, 175; 31 \#3815.

Tata Institute of Fundamental Research, Bombay 400005, India 